\title{
FHA Domain-Ligand Interactions: Importance of Integrating Chemical and Biological Approaches
}

Anjali Mahajan, ${ }^{1}$ Chunhua Yuan, ${ }^{2}$ Brietta L. Pike, ${ }^{3}$ Jörg Heierhorst, ${ }^{3}$ Chi-Fon Chang, ${ }^{4}$ and Ming-Daw

Tsai $^{1,2,4,5^{\circ}}$

${ }^{1}$ Biophysics Program, ${ }^{2}$ Campus Chemical Instrument Center, ${ }^{5}$ Departments of Chemistry and Biochemistry, The Ohio State University, Columbus, OH 43210, USA; ${ }^{3}$ Molecular Genetics Unit, St. Vincent's Institute of Medical Research, and Department of Medicine SVH, University of Melbourne, Melbourne, Australia; ${ }^{4}$ Genomics Research Center, Academia Sinica, Taipei, Taiwan.

RECEIVED DATE (automatically inserted by publisher); Tsai.7@osu.edu

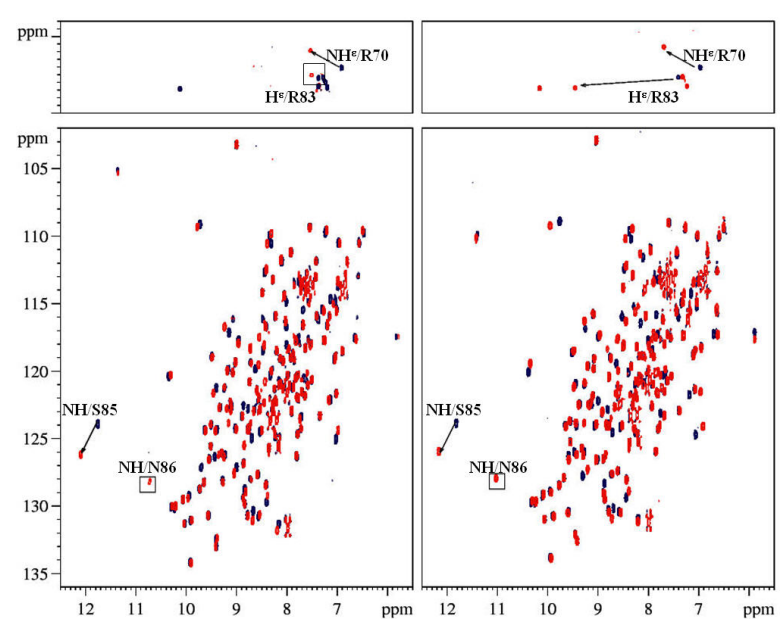

(a) FHA1-pT(Mdt1)

(b) FHA1-pT(Rad9)

Figure 1: ${ }^{1} \mathrm{H}^{15} \mathrm{~N}$ HSQC spectra showing peptide binding induced perturbations: (a) FHA1-pT(Mdt1); (b) FHA1-pT(Rad9).

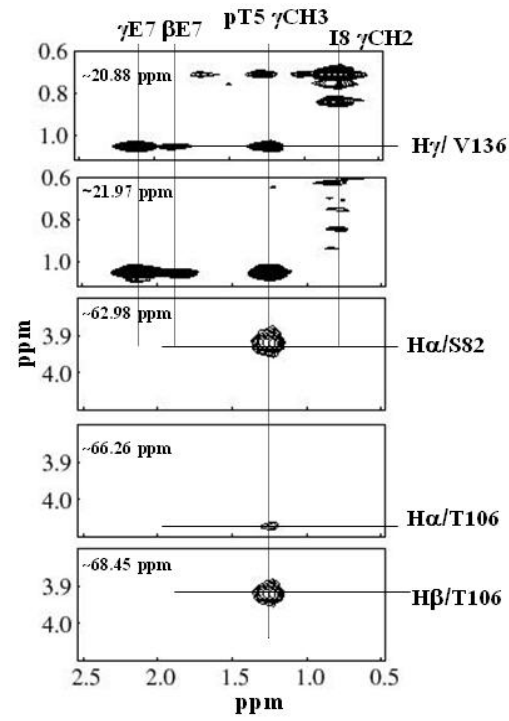

Figure 2: Selected regions of the $3 \mathrm{D}{ }^{13} \mathrm{C}$-edited $(f 1),{ }^{13} \mathrm{C} /{ }^{15} \mathrm{~N}$-filtered $(f 3)$ NOESY spectrum.

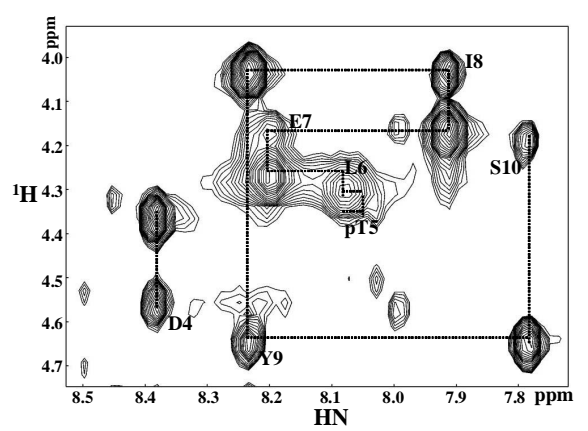

Figure 3: A portion of $2 \mathrm{D}{ }^{13} \mathrm{C} /{ }^{15} \mathrm{~N}$-filtered NOESY in $\mathrm{H}_{2} \mathrm{O}$ depicting strong $\mathrm{H}^{\alpha}(\mathrm{i})-\mathrm{H}^{\mathrm{N}}(\mathrm{i}+1)$ sequential NOEs of peptide.
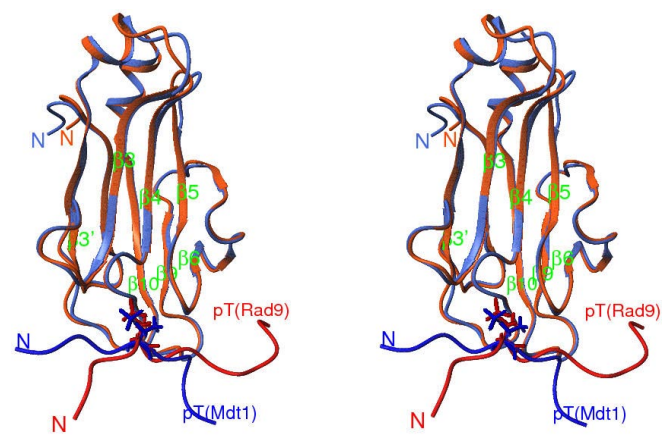

Figure 4: Ribbon diagram of the FHA1-pT(Mdt1) complex overlaid with that of the FHA1-pT(Rad9) complex obtained previously. ${ }^{1}$
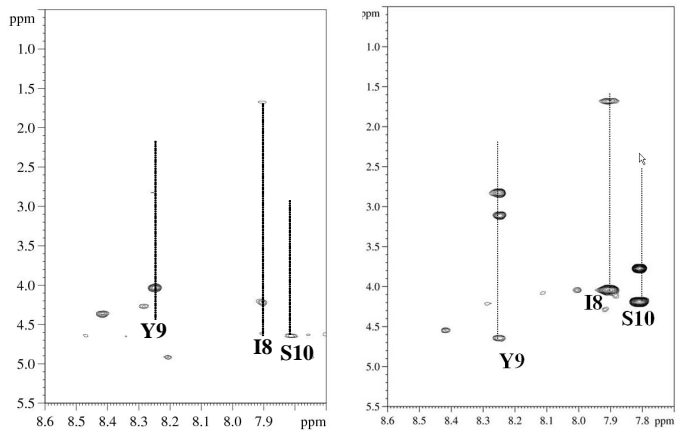

Figure 5: Protection of amide protons of $\mathrm{I}(\mathrm{pT}+3), \mathrm{Y}(\mathrm{pT}+4)$ and $\mathrm{S}(\mathrm{pT}+5)$ observed in $2 \mathrm{D}{ }^{13} \mathrm{C} /{ }^{15} \mathrm{~N}$-filtered NOESY (left) and TOCSY (right) collected in $\mathrm{D}_{2} \mathrm{O}$ for peptide assignments. 


\begin{tabular}{|c|c|}
\hline \multicolumn{2}{|l|}{ NMR distance \& dihedral constraints } \\
\hline Total distance constraints & 2524 \\
\hline FHA1 NOE & 2116 \\
\hline Mdt1(301-310)1P NOE & 97 \\
\hline FHA1-Mdt1(301-310)1P intermolecular $\mathrm{NOE}^{\mathrm{c}}$ & 41 \\
\hline FHA1 H-bonds & $39 \times 2$ \\
\hline Total dihedral angle constraints & 192 \\
\hline FHA $1 \phi^{a}$ & 96 \\
\hline \multicolumn{2}{|l|}{ Structure statistics (20 structures) } \\
\hline \multicolumn{2}{|l|}{ Violation statistics } \\
\hline Deviations from idealized covalent geometry & $0.029 \pm 0.000$ \\
\hline Bond lengths $(\AA)$ & $0.0036 \pm 0.00001$ \\
\hline Bond angles $\left({ }^{\circ}\right)$ & $0.446 \pm 0.005$ \\
\hline Impropers $\left({ }^{\circ}\right)$ & $0.332 \pm 0.009$ \\
\hline \multicolumn{2}{|l|}{ R.m.s. deviations from the average structure $(\AA)^{b}$} \\
\hline Backbone atoms (residues 28-51, 63-157) & $0.46 \pm 0.04$ \\
\hline All heavy atoms (residues $28-51,63-157$ ) & $0.90 \pm 0.06$ \\
\hline \multicolumn{2}{|l|}{ PROCHECK (Ramachandran plot) } \\
\hline Most favored and additionally allowed regions (\%) & $95.5 \pm 1.5$ \\
\hline
\end{tabular}

${ }^{a} \phi$ and $\psi$ constraints were derived by using TALOS ${ }^{2}$.

${ }^{b}$ R.m.s. deviations from the average structure were calculated for the final 22 structures by excluding poorly defined regions (FHA1 residues 51-63).

${ }^{\mathrm{c}}$ Two artificial distance restraints are included: $\mathrm{C}^{\zeta}$ of $\mathrm{R} 70$ to the $\mathrm{P}$ atom of $\mathrm{pT}$ and $\mathrm{C}^{\gamma} \mathrm{of} \mathrm{N} 86$ to the $\mathrm{P}$ atom of pT.

Table 2. Intermolecular NOEs assigned in FHA1-pT(Mdt1) complex

\begin{tabular}{|c|c|c|c|}
\hline Protons & Intensity & Protons & Intensity \\
\hline $\mathrm{H}^{\alpha} / \mathrm{S} 82-\mathrm{C}^{\beta} / \mathrm{L}(\mathrm{pT}+1)$ & Weak & $\mathrm{H}^{\gamma / 2} / \mathrm{T} 106-\mathrm{H}^{\gamma_{1}} / \mathrm{E}(\mathrm{pT}+2)$ & Medium \\
\hline $\mathrm{H}^{\alpha} / \mathrm{S} 82-\mathrm{H}^{\gamma} / \mathrm{L}(\mathrm{pT}+1)$ & Very Weak & $\mathrm{H}^{\gamma / 2} / \mathrm{T} 106-\mathrm{H}^{\gamma / 2} / \mathrm{E}(\mathrm{pT}+2)$ & Medium \\
\hline $\mathrm{H}^{\alpha} / \mathrm{S} 82-\mathrm{H}^{\delta_{1}} / \mathrm{L}(\mathrm{pT}+1)$ & Medium & $\mathrm{H}^{\beta 1} / \mathrm{N} 107-\mathrm{H}^{\gamma} / \mathrm{L}(\mathrm{pT}+1)$ & Very Weak \\
\hline $\mathrm{H}^{\alpha} / \mathrm{S} 82-\mathrm{H}^{\delta 2} / \mathrm{L}(\mathrm{pT}+1)$ & Medium & $\mathrm{H}^{\beta 2} / \mathrm{N} 107-\mathrm{H}^{\gamma} / \mathrm{L}(\mathrm{pT}+1)$ & Very Weak \\
\hline $\mathrm{H}^{\beta} / \mathrm{S} 82-\mathrm{H}^{\beta \gamma} / \mathrm{L}(\mathrm{pT}+1)$ & Medium & $\mathrm{H}^{\alpha 1} / \mathrm{G} 135-\mathrm{H}^{\delta_{1}} / \mathrm{I}(\mathrm{pT}+3)$ & Weak \\
\hline $\mathrm{H}^{\beta} / \mathrm{S} 82-\mathrm{H}^{\delta_{1}} / \mathrm{L}(\mathrm{pT}+1)$ & Medium & $\mathrm{H}^{\alpha 2} / \mathrm{G} 135-\mathrm{H}^{\delta_{1}} / \mathrm{I}(\mathrm{pT}+3)$ & Weak \\
\hline $\mathrm{H}^{\beta} / \mathrm{S} 82-\mathrm{H}^{\delta 2} / \mathrm{L}(\mathrm{pT}+1)$ & Medium & $\mathrm{H}^{\alpha} / \mathrm{V} 136-\mathrm{H}^{\delta_{1}} / \mathrm{I}(\mathrm{pT}+3)$ & Very Weak \\
\hline $\mathrm{H}^{\beta_{1}} / \mathrm{R} 83-\mathrm{H}^{\delta} / \mathrm{L}(\mathrm{pT}+1)$ & Weak & $\mathrm{H}^{\beta} / \mathrm{V} 136-\mathrm{H}^{\delta_{1}} / \mathrm{I}(\mathrm{pT}+3)$ & Very Weak \\
\hline $\mathrm{H}^{\beta 2} / \mathrm{R} 83-\mathrm{H}^{\delta} / \mathrm{L}(\mathrm{pT}+1)$ & Weak & $\mathrm{H}^{\gamma 1} / \mathrm{V} 136-\mathrm{H}^{\alpha} / \mathrm{I}(\mathrm{pT}+3)$ & Weak \\
\hline $\mathrm{H}^{\beta} / \mathrm{R} 83-\mathrm{H}^{\beta \gamma} / \mathrm{L}(\mathrm{pT}+1)$ & Very Weak & $\mathrm{H}^{\gamma /} / \mathrm{V} 136-\mathrm{H}^{\beta} / \mathrm{I}(\mathrm{pT}+3)$ & Weak \\
\hline $\mathrm{H}^{\delta_{1}} / \mathrm{R} 83-\mathrm{H}^{\delta} / \mathrm{L}(\mathrm{pT}+1)$ & Weak & $\mathrm{H}^{\gamma_{1}} / \mathrm{V} 136-\mathrm{H}^{\gamma_{1}} / \mathrm{I}(\mathrm{pT}+3)$ & Medium \\
\hline $\mathrm{H}^{\delta 2} / \mathrm{R} 83-\mathrm{H}^{\delta} / \mathrm{L}(\mathrm{pT}+1)$ & Weak & $\mathrm{H}^{\gamma 1} / \mathrm{V} 136-\mathrm{H}^{\gamma 2} / \mathrm{I}(\mathrm{pT}+3)$ & Weak \\
\hline $\mathrm{H}^{\alpha} / \mathrm{S} 85-\mathrm{H}^{\gamma^{2}} / \mathrm{pT}$ & Medium & $\mathrm{H}^{\gamma_{1}} / \mathrm{V} 136-\mathrm{H}^{\delta_{1}} / \mathrm{I}(\mathrm{pT}+3)$ & Strong \\
\hline $\mathrm{H}^{\alpha} / \mathrm{T} 106-\mathrm{H}^{\gamma / 2} / \mathrm{pT}$ & Weak & $\mathrm{H}^{1 / 2} / \mathrm{V} 136-\mathrm{H}^{12} / \mathrm{I}(\mathrm{pT}+3)$ & Very Weak \\
\hline $\mathrm{H}^{\beta} / \mathrm{T} 106-\mathrm{H}^{1 / 2} / \mathrm{pT}$ & Medium & $\mathrm{H}^{\gamma / 2} / \mathrm{V} 136-\mathrm{H}^{\delta_{1}} / \mathrm{I}(\mathrm{pT}+3)$ & Weak \\
\hline $\mathrm{H}^{1 / 2} / \mathrm{T} 106-\mathrm{H}^{\beta} / \mathrm{pT}$ & Weak & $\mathrm{H}^{\gamma /} / \mathrm{V} 136-\mathrm{H}^{\delta} / \mathrm{Y}(\mathrm{pT}+4)$ & Medium \\
\hline $\mathrm{H}^{1 / 2} / \mathrm{T} 106-\mathrm{H}^{1 / 2} / \mathrm{pT}$ & Medium & $\mathrm{H}^{\gamma /} / \mathrm{V} 136-\mathrm{H}^{\varepsilon} / \mathrm{Y}(\mathrm{pT}+4)$ & Medium \\
\hline $\mathrm{H}^{\gamma / 2} / \mathrm{T} 106-\mathrm{H}^{\alpha} / \mathrm{E}(\mathrm{pT}+2)$ & Very Weak & $\mathrm{H}^{\gamma / 2} / \mathrm{V} 136-\mathrm{H}^{\delta} / \mathrm{Y}(\mathrm{pT}+4)$ & Medium \\
\hline $\mathrm{H}^{\gamma_{2}} / \mathrm{T} 106-\mathrm{H}^{\beta_{1}} / \mathrm{E}(\mathrm{pT}+2)$ & Weak & $\mathrm{H}^{12} / \mathrm{V} 136-\mathrm{H}^{\varepsilon} / \mathrm{Y}(\mathrm{pT}+4)$ & Medium \\
\hline $\mathrm{H}^{1 / 2} / \mathrm{T} 106-\mathrm{H}^{\beta 2} / \mathrm{E}(\mathrm{pT}+2)$ & Weak & & \\
\hline
\end{tabular}

\section{Materials and methods}

Sample preparation: ${ }^{13} \mathrm{C} /{ }^{15} \mathrm{~N}$-labeled $\mathrm{FHA} 1$ samples were purified as described ${ }^{3}$. The non-labeled peptide was purchased from Genemed synthesis Inc., CA.

NMR experiments: NMR experiments were performed on Bruker DRX-800, AV800 and AV500 at 293K. $2 \mathrm{D}{ }^{3} \mathrm{C} /{ }^{15} \mathrm{~N}-$ filtered NOESY, COSY and TOCSY experiments were performed in $\mathrm{H}_{2} \mathrm{O}$ as well as in $\mathrm{D}_{2} \mathrm{O}$ for the peptide assignments. 3D ${ }^{13} \mathrm{C}$-edited $(f 1),{ }^{13} \mathrm{C} /{ }^{15} \mathrm{~N}$-filtered $(f 3)$ NOESY in $90 \%$ $\mathrm{H}_{2} \mathrm{O} / 10 \% \mathrm{D}_{2} \mathrm{O}$ and ${ }^{13} \mathrm{C} /{ }^{15} \mathrm{~N}$-filtered $(f 1),{ }^{13} \mathrm{C}$-edited $(f 3) \mathrm{NOESY}$ in $100 \% \mathrm{D}_{2} \mathrm{O}$ were conducted to extract the intermolecular restraints. $3 \mathrm{D}{ }^{13} \mathrm{C}$-edited NOESY was also collected on the complex to assist the intermolecular NOE assignments.

Structural calculation: Structures were generated by simulated annealing method using $\mathrm{CNS}^{4}$, and analyzed by PROCHECK $^{5}$ and MOLMOL. ${ }^{6}$
1. Yuan, C.; Yongkiettrakul, S.; Byeon, I. J.; Zhou, S.; Tsai, M. D., Solution structures of two FHA1-phosphothreonine peptide complexes provide insight into the structural basis of the ligand specificity of FHA1 from yeast Rad53. J Mol Biol 2001, 314, (3), 563-75.

2. Cornilescu, G.; Delaglio, F.; Bax, A., Protein backbone angle restraints from searching a database for chemical shift and sequence homology. J. Biomol. NMR 1999, 13, (3), 289-302.

3. Liao, H.; Yuan, C.; Su, M. I.; Yongkiettrakul, S.; Qin, D.; Li, H. Byeon, I. J.; Pei, D.; Tsai, M. D., Structure of the FHA1 domain of yeast Rad53 and identification of binding sites for both FHA1 and its target protein Rad9. J Mol Biol 2000, 304, (5), 941-51.

4. Brunger, A. T.; Adams, P. D.; Clore, G. M.; DeLano, W. L.; Gros, P.; Grosse-Kunstleve, R. W.; Jiang, J. S.; Kuszewski, J.; Nilges, M.; Pannu, N. S.; Read, R. J.; Rice, L. M.; Simonson, T.; Warren, G. L., Crystallography \& NMR system: A new software suite for macromolecular structure determination. Acta Crystallogr D Biol Crystallogr 1998, 54 (Pt 5), 905-21.

5. Laskowski, R. A.; Macarthur, M. W.; Moss, D. S.; Thornton, J. M. PROCHECK - a program to check the stereochemical quality of protein structures. J. Appl. Crystallogr. 1993, 26, 283-291.

6. Koradi, R.; Billeter, M.; Wuthrich, K., MOLMOL: a program for display and analysis of macromolecular structures. J. Mol. Graph. $1996,14,51-55$. 